Leistritz, Lutz; Putsche, Peter; Schwab, Karin; Hesse, Wolfram; Süße, Thomas;

Haueisen, Jens; Witte, Herbert:

Coupled oscillators for modeling and analysis of EEG/MEG oscillations

Zuerst erschienen in: Biomedizinische Technik = Biomedical Engineering. - Berlin [u.a.] : de Gruyter. - 52 (2007), 1, p. 83-89.

Erstveröffentlichung: $\quad$ 2007-02-22

ISSN (online): $\quad$ 1862-278X

ISSN (print): $\quad$ 0013-5585

DOI: $\quad$ 10.1515/BMT.2007.016

[Zuletzt gesehen: 43696]

„Im Rahmen der hochschulweiten Open-Access-Strategie für die Zweitveröffentlichung identifiziert durch die Universitätsbibliothek IImenau. “

"Within the academic Open Access Strategy identified for deposition by Ilmenau University Library."

„Dieser Beitrag ist mit Zustimmung des Rechteinhabers aufgrund einer (DFGgeförderten) Allianz- bzw. Nationallizenz frei zugänglich."

"This publication is with permission of the rights owner freely accessible due to an Alliance licence and a national licence (funded by the DFG, German

DFG

Research Foundation) respectively." 


\section{Coupled oscillators for modeling and analysis of EEG/MEG oscillations}

\author{
Lutz Leistritz ${ }^{1, \star}$, Peter Putsche1, Karin Schwab1, \\ Wolfram Hesse ${ }^{1}$, Thomas Süße ${ }^{1}$, Jens \\ Haueisen $^{2,3}$ and Herbert Witte ${ }^{1}$ \\ ${ }^{1}$ Institute of Medical Statistics, Computer Sciences and \\ Documentation, Medical Faculty, Friedrich Schiller \\ University Jena, Jena, Germany \\ 2 Biomagnetic Center, Department of Neurology, \\ Medical Faculty, Friedrich Schiller University Jena, \\ Jena, Germany \\ ${ }^{3}$ Institute of Biomedical Engineering and Informatics, \\ Technical University IImenau, IImenau, Germany
}

\begin{abstract}
This study presents three EEG/MEG applications in which the modeling of oscillatory signal components offers complementary analysis and an improved explanation of the underlying generator structures. Coupled oscillator networks were used for modeling. Parameters of the corresponding ordinary coupled differential equation (ODE) system are identified using EEG/MEG data and the resulting solution yields the modeled signals. This model-related analysis strategy provides information about the coupling quantity and quality between signal components (example 1, neonatal EEG during quiet sleep), allows identification of the possible contribution of hidden generator structures (example 2, 600-Hz MEG oscillations in somatosensory evoked magnetic fields), and can explain complex signal characteristics such as amplitude-frequency coupling and frequency entrainment (example 3, EEG burst patterns in sedated patients).
\end{abstract}

Keywords: coupled oscillators; EEG; MEG; model-based signal analysis; parameter identification.

\section{Introduction}

EEG/MEG oscillations show characteristics identified in experiments using simulations with coupled oscillators, such as linear and non-linear phase synchronization/desynchronization of the oscillations and frequency entrainment [3]. As already demonstrated by other studies, simulations of physiological systems using coupled oscillator systems and corresponding model-based analysis methods not only contribute significantly to improve interpretation of the results, but also enhance the analysis methods themselves [4, 24]. In addition, coupled

*Corresponding author: Lutz Leistritz, Institute of Medical

Statistics, Computer Sciences and Documentation,

Bachstr. 18, 07740 Jena, Germany

Phone: +49-3641-93405

Fax: +49-3641-933200

E-mail: lutz.leistritz@mti.uni-jena.de oscillators (oscillator networks) can be directly used as an analysis method, whereby the parameters of the underlying ordinary coupled differential equation (ODE) system are identified using the measured EEG/MEG data [13]. With this type of adapted ODE system, the solution yields the modeled signals. Using one ODE (oscillator), one EEG/MEG signal or signal component can be modeled. Interrelations between the signals or signal components are modeled by couplings between ODEs [22].

The aim of this study is to demonstrate that modelbased approaches can be used to analyze EEG patterns (example 1, neonatal EEG during quiet sleep). It can also be shown that models of coupled oscillators allow the identification of influences of hidden sources that cannot be measured and for which the signal characteristics are unknown. Adding hidden sources to the model leads to improved modeling and explanation of the EEG/MEG signal results (example 2, 600-Hz MEG oscillations in somatosensory evoked magnetic fields). Furthermore, both approaches can be combined. A signal component related to a hidden source (e.g., known from experimental studies) can be used to model the contribution of such a hidden source to the whole signal pattern and to its signal characteristics. Parameters of a complete model might be utilized as analysis parameters (example 3, EEG burst patterns in sedated patients).

\section{Materials and methods}

\section{Example 1: oscillatory networks for coupling analysis}

The first example is related to a group of six clinically and neurologically normal, full-term neonates (mean conceptual age 39.3 weeks, range 38-41; mean birth weight 3152 g, range 2670-3420; 5-min APGAR score $\geq 8$ ). The recordings were performed during sleep between 09:00 and 12:00 h. The EEG (unipolar recordings with linked ear reference; 8-channel EEG; international 10-20 systems with electrodes Fp1, Fp2, C3, C4, T3, T4, O1, O2; sampling rate $128 \mathrm{~Hz}$ ), heart rate, respiratory movements and EOG were recorded. Only the EEG recordings during quiet sleep were selected.

The EEG was classified by a trained physician into the burst and interburst patterns. The data were filtered using two FFT filters $(1.0-1.6$ and $3.5-6.0 \mathrm{~Hz})$ to extract relevant frequency ranges. Signal segments of $6 s$ in duration were used for the present examination, whereby one segment consists of a consecutive series of a 1-s interburst interval followed by a 4-s burst period and again a 1-s interburst.

\section{Example 2: modeling to explain $600-\mathrm{Hz}$ oscillations}

Somatosensory evoked potentials and magnetic fields were simultaneously derived from 10 healthy volunteers 
after electrical stimulation of the nervus medianus (7000 averages, sampling frequency $5 \mathrm{kHz}$, anti-aliasing lowpass filter $1500 \mathrm{~Hz}$ ). In addition, a 3D MRI data set was acquired from each volunteer $\left(1-\mathrm{mm}^{3}\right.$ voxel size; $256 \times 256 \times 192)$. The measurement was performed with 31 magnetic channels (Philips, Hamburg, Germany) and 32 electrical channels (Neuroscan, El Paso, TX, USA). Stimulation involved rectangular electrical impulses of $0.2 \mathrm{~ms}$ in duration at a frequency of $4 \mathrm{~Hz}$. To improve the signal/noise ratio, the registered data were filtered offline using an optimal filter (Wiener filter) and were also digitally filtered by a third-order Butterworth filter with a band of $450-750 \mathrm{~Hz}$. Then singular value decomposition was performed and the noise proportion was eliminated. Electrical and magnetic data were used simultaneously for source reconstruction. The 3D MRI data for the ten volunteers were used to compute an individual head model consisting of the three components skin, cranium and brain. The individual head models were used for subsequent source localization. The locations of Brodmann areas $3 b$ and 1 were identified by fitting a 2-dipole model using the Nelder-Mead simplex algorithm.

\section{Example 3: coupled Duffing oscillators to model and analyze amplitude-frequency dependence and frequency entrainment}

Investigations were carried out on seven sedated patients with various neurosurgical diseases, a subgroup (thiopental group) of a group of patients who have been described in detail elsewhere [27]. All were given individualized basic sedation to ensure controlled mechanical ventilation and safe nursing. An increase in intracranial pressure that could not be influenced by conventional therapy (ventricular drainage if available, hyperventilation to a $\mathrm{PaCO}_{2}$ of $<4.6 \mathrm{kPa}$ and administration of mannitol) was used as the indicator to increase basic sedation by adding i.v. hypnotics (thiopental in our group). The EEG was recorded by an eight-channel analogous EEG (Neurofax 5610G, Nihon Kohden, Tokyo, Japan); the electrodes were placed in superior lengthwise positions according to the international $10-20$ system with $\mathrm{Cz}$ as the reference. This method is the favored recording mode in important clinical studies. The sampling frequency was $256 \mathrm{~Hz}$. Down-sampling to a rate of $128 \mathrm{~Hz}$ was performed after digital low-pass filtering with an upper cutoff frequency of $64 \mathrm{~Hz}$. Only the first $2 \mathrm{~s}$ of the EEG patterns were used for analysis (256 sampling points after downsampling), with the underlying models fitted to these burst sections of a duration of $2 \mathrm{~s}$.

The burst onset positions were automatically detected by the pattern detection unit developed by Leistritz et al. [12] on the basis of Fp1 vs. Cz.

\section{Results}

\section{Example 1: oscillatory networks for coupling analysis}

The field of application of coupled oscillators includes the analysis of multidimensional signals with different and unknown interactions between the signal components.
This requires structuring of a network of coupled oscillators, which includes definitions of the oscillator types and possible couplings between the network oscillators.

The goal of this example was to fit oscillatory networks of coupled damped harmonic oscillators to burst segments of eight-channel EEG recordings of healthy neonates. A fully connected network would contain 56 possible couplings, too many for reliable parameter identification. A reasonable possibility to reduce this degree of freedom is to assume that only couplings between adjacent electrode positions are admissible. This results in a reduction to 34 couplings.

We assumed a linear model

$\ddot{x}(t)=\operatorname{diag}(\boldsymbol{\mu}) \dot{x}-\operatorname{diag}(2 \pi \boldsymbol{\omega})^{2} x+(E-I) x$, $x(0)=x_{0}, \quad \dot{x}(0)=\chi_{0}$,

with frequency and damping parameters $\boldsymbol{\omega}=$ $\left(\omega_{1}, \omega_{2}, \ldots, \omega_{8}\right)$ and $\boldsymbol{\mu}=\left(\mu_{1}, \mu_{2}, \ldots, \mu_{8}\right)$ and as the coupling matrix

$$
E=\left(\begin{array}{cccccccc}
0 & \varepsilon_{1} & \varepsilon_{2} & \varepsilon_{3} & \varepsilon_{4} & 0 & 0 & 0 \\
\varepsilon^{\prime}{ }_{1} & 0 & \varepsilon_{5} & \varepsilon_{6} & 0 & \varepsilon_{7} & 0 & 0 \\
\varepsilon_{2}^{\prime} & \varepsilon_{5}^{\prime} & 0 & \varepsilon_{8} & \varepsilon_{9} & 0 & \varepsilon_{10} & \varepsilon_{11} \\
\varepsilon^{\prime}{ }_{3} & \varepsilon^{\prime}{ }_{6} & \varepsilon^{\prime}{ }_{8} & 0 & 0 & \varepsilon_{12} & \varepsilon_{13} & \varepsilon_{14} \\
\varepsilon^{\prime}{ }_{4} & 0 & \varepsilon^{\prime}{ }_{9} & 0 & 0 & 0 & \varepsilon_{15} & 0 \\
0 & \varepsilon^{\prime}{ }_{7} & 0 & \varepsilon^{\prime}{ }_{12} & 0 & 0 & 0 & \varepsilon_{16} \\
0 & 0 & \varepsilon_{10}^{\prime} & \varepsilon^{\prime}{ }_{13} & \varepsilon^{\prime}{ }_{15} & 0 & 0 & \varepsilon_{17} \\
0 & 0 & \varepsilon^{\prime}{ }_{11} & \varepsilon^{\prime}{ }_{14} & 0 & \varepsilon^{\prime}{ }_{16} & \varepsilon^{\prime}{ }_{17} & 0
\end{array}\right) .
$$

Altogether, a system with 66 unknown parameters 34 coupling parameters, eight damping factors, eight frequency parameters and 16 initial conditions - had to be identified. Separate oscillatory network models were fitted for each frequency range using Bock's multiple shooting method [2] with the underlying modified Levenberg-Marquardt method [15, 16]. To reduce the influence of different amplitudes at different electrode positions, the data were normalized to a maximum norm of one. Figure 1 depicts the results for one fit. Typically, we could reach a better fit at the frontal electrode positions Fp1 and Fp2 in comparison to the occipital positions. It was more difficult to find an appropriate minimum of the error function using data sets of the higher frequency range. Besides the possibility of an inadequate model, another possible explanation might be the much more complicated structure of the error function, which is more "furrowed" in this case. Consequently, the dependence of the optimal parameter set on the initialization of the optimization procedure is increased. The quality of the fit typically decreases from frontal to occipital positions and from the lower to the higher frequency range. Our previous studies have demonstrated that the synchronization properties (coherence and quadratic phase couplings $[5,23])$ are significantly pronounced at the frontal electrodes. 

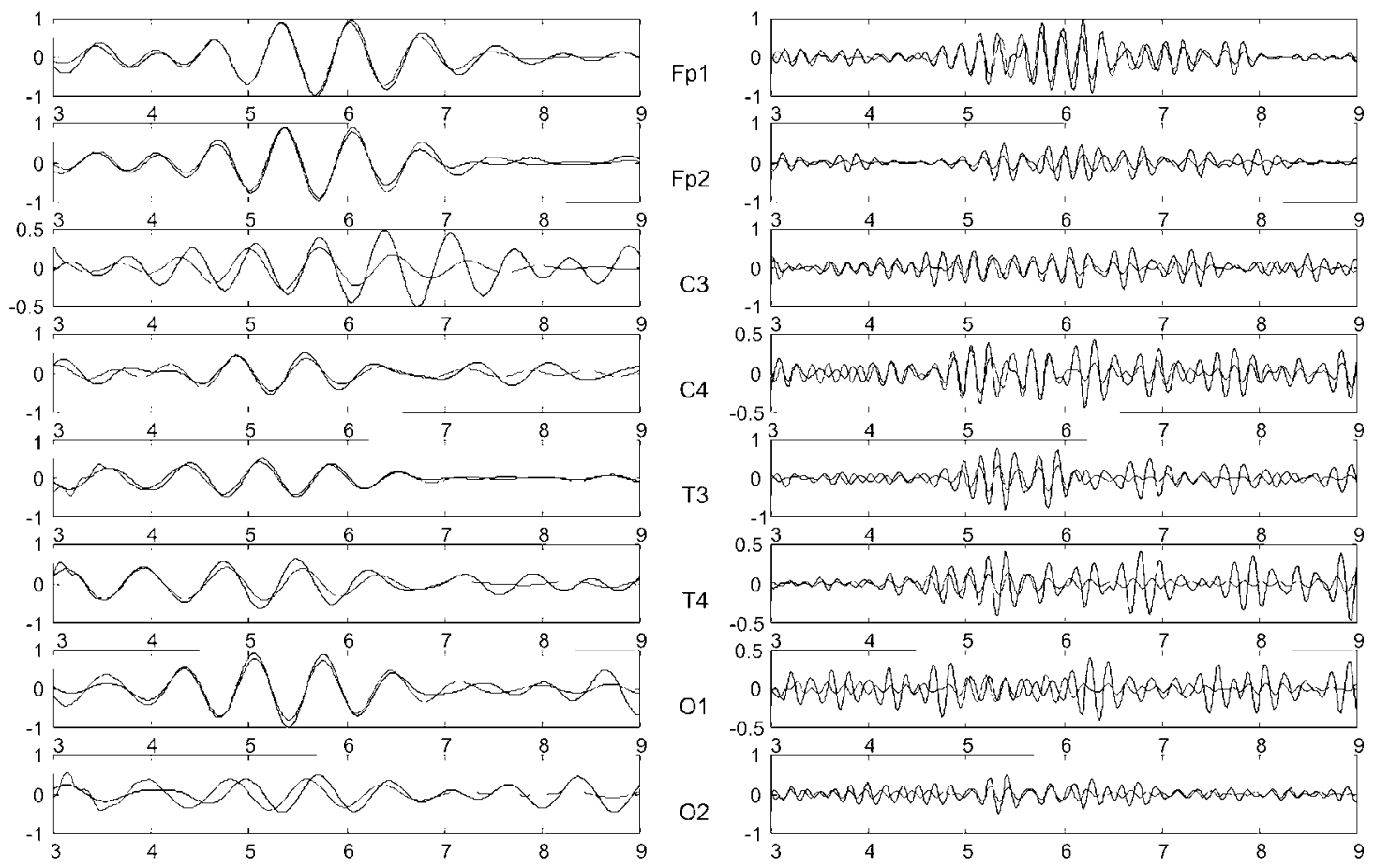

Figure 1 Optimization results for the lower frequency range 1.0-1.6 Hz (left) and the higher frequency range $3.5-6.0 \mathrm{~Hz}$ (right). The dashed lines represent the measured data and the solid lines depict the fitted model functions.

\section{Example 2: modeling to explain $600-\mathrm{Hz}$ oscillations}

Directed information transfer in the human brain presumably occurs by oscillations $[10,19]$. The second example demonstrates the possibility of modeling cortical $600-\mathrm{Hz}$ oscillations localized within Brodmann areas $3 \mathrm{~b}$ and 1 after stimulation of the nervus medianus using a system of coupled differential equations. The origin and function of these $600-\mathrm{Hz}$ activities are not completely understood yet, but a causal connection seems likely. The aim of the present example was to derive a fairly uncomplicated model to describe the spatial-temporal features of these $600-\mathrm{Hz}$ activities. The model may be used to analyze the directed information transfer of the somatosensory system. In fact, once a model is established and fitted to real data, explorative analyses may be performed to investigate what transpires when single parameters of the system are slightly changed or couplings between the network oscillators are cancelled. Such modifications could offer a significant contribution to the understanding of network properties.

Basically, there are two key points that have to be considered to solve such types of task. The first involves determination of the model in combination with the definition of free parameters that have to be optimized. The second is selection of a suitable optimization procedure that can identify a reliable parameter set that minimizes a certain error function, and that provides a good model fit. The activation time courses of the $600-\mathrm{Hz}$ activity formed the observation data for fitting of the oscillatory network models.

The following assumptions had to be embedded into a model for the cortical and thalamic $600-\mathrm{Hz}$ activity:

1. The cortical activities are initiated from a resting state of zero by a thalamic impulse resulting from irritation of the nervus medianus;
2. The thalamic component may be formalized by an oscillation with amplitude modulated by a Gaussian shape function [7, 8];

3. The cortical components can be modeled by damped harmonic oscillators that have a mutual influence;

4. Due to the sequential activation of different brain areas, impulse propagation is delayed from the thalamic component to area $3 \mathrm{~b}$ (1.2 ms fixed) and to area 1 (variable, approx. $2.4 \mathrm{~ms}$ ); and

5. The state before stimulation is assumed to be zero.

Denoting Brodmann area $3 b$ by $x$, area 1 by $y$ and the thalamic component by $z$, the following differential algebraic equation results:

$$
\begin{aligned}
& \ddot{x}=\mu_{1} \dot{x}+\varepsilon_{11} x+\varepsilon_{12} y+\varepsilon_{13} z(t-0.0012), \\
& \ddot{y}=\mu_{2} \dot{x}+\varepsilon_{21} x+\varepsilon_{22} y+\varepsilon_{23} z(t-\delta), \\
& z(t)=e^{-\frac{(t-\mu)^{2}}{2 \sigma^{2}}} \cdot \cos (2 \pi \omega t+\varphi)
\end{aligned}
$$

with $x(0)=\dot{x}(0)=y(0)=\dot{y}(0)=0$. All assumptions are inserted into a model that contains 13 parameters $\left(\mu, \mu_{1}, \mu_{2}, \sigma, \varpi, \varphi, \varepsilon_{11}, \varepsilon_{12}, \varepsilon_{13}, \varepsilon_{21}, \varepsilon_{22}, \varepsilon_{23}, \delta\right)$, where the time delay $\delta$ was limited by the bounds 2.2 and $2.7 \mathrm{~ms}$, which are reasonable physiological limits. The parameter set may be considered as minimal, since any elimination would result in a violation of at least one of the assumptions above. Since $x(0)=\dot{x}(0)=y(0)=\dot{y}(0)=0$, the thalamic component is necessary to push the cortical components off their resting state of zero.

This model was fitted to all data sets separately using the least-square deviation from the model to the measured data points as the error criterion. The fitting process is not trivial, since the error function possesses a multitude of local minima. There are different strategies to 
reduce the influence of the local convergence of optimization procedures, such as a combination of deterministic and stochastic search algorithms [16, 20], Bock's multiple shooting method [2], or simulated annealing [11]. We alternately used Bock's multiple shooting and simulated annealing until no further improvement in the approximation quality could be achieved.

The differential algebraic model could be fitted successfully for all ten data sets. As an example, the model functions with the embedded data points are depicted in Figure 2, where the best and worst approximations for larger data sets were chosen. The thalamic component $z$ is also given to demonstrate the amplitude-modulated oscillating structure of this component. Although the single parameters vary from volunteer to volunteer, the coupling parameters $\varepsilon_{*}$ did not vanish for all data sets. This is an indication that the underlying oscillatory network topology is equal for all volunteers. We could show that cortical $600-\mathrm{Hz}$ activity may be modeled by a system of three coupled oscillators.

\section{Example 3: coupled Duffing oscillators to model and analyze amplitude-frequency dependence and frequency entrainment}

As shown in previous studies, two dominant oscillatory components of EEG burst patterns during electroencephalographic burst suppression periods (BSPs) exist in sedated intensive care patients [17, 25]. A low-frequency oscillation $(<5 \mathrm{~Hz})$, which starts with an initial negative wave, is superimposed by a delayed spindle-like highfrequency oscillation (>8 Hz; high frequency). These are characterized by a transient but strong quadratic-phase coupling (QPC) [18]. QPC can be demonstrated between the frequency ranges $0-2.5 \mathrm{~Hz}$ and $8-12 \mathrm{~Hz}(3-7.5 \mathrm{~Hz})$ initiated $0.25 \mathrm{~s}$ after burst onset and reaches a maximum between 0.75 and $1.25 \mathrm{~s}$. The most probable cause of the QPC was identified as an amplitude modulation, i.e., the low-frequency oscillation modulates the amplitude of the high-frequency oscillation. This model has been evaluated by a special amplitude demodulation method [1, 26]. However, not all coupling properties can be
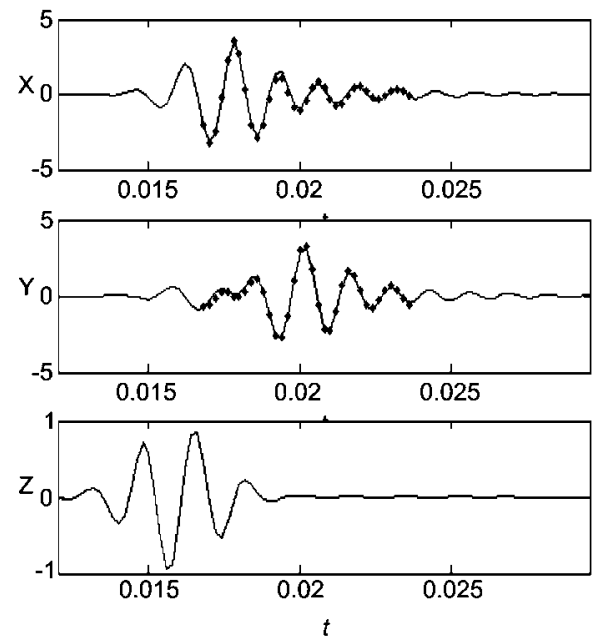

explained by amplitude modulation. It can be shown that an amplitude-frequency dependence of both frequency bands exists. An alternative interpretation of the amplitude-frequency dependence is amplitude and frequency entrainment, i.e., the amplitude of the low-frequency band entrains the amplitude of the high-frequency band, and the frequencies of both bands are also coupled. Coupled Duffing oscillators are used to explain these coupling characteristics and the time courses of amplitude and frequency modulation. Modeling was performed on the basis of the following presumptions:

1. The high-frequency component was taken to model a thalamic oscillator that mutually interacts with the low-frequency cortical oscillatory activity;

2. The high-frequency component has a spindle-like amplitude;

3. The frequency of the high-frequency component may be dependent on the amplitude;

4. The low-frequency component is modeled by a damped oscillator; and

5. The frequency of the low-frequency component may be dependent on the amplitude.

Thus, a suitable model for the low-frequency cortical oscillatory activity is the damped Duffing oscillator given by the ODE

$$
\begin{aligned}
& \ddot{x}=-\left(2 \pi \omega_{L}\right)^{2} x-\mu \dot{x}-\beta_{L} x^{3} \\
& x(0)=x_{0}, \quad \dot{x}(0)=\chi_{0} .
\end{aligned}
$$

Furthermore, the ODE

$\ddot{y}=-\left(\left(2 \pi \omega_{H}\right)^{2}+\frac{2}{\tau}+4\left(\frac{\left(t-t_{0}\right)^{2}}{\tau}\right)\right) y-\frac{t-t_{0}}{\tau} \dot{y}$

has the solution

$$
y(t)=a \cdot \cos \left(2 \pi \omega_{H} t+\varphi\right) \cdot e^{-\frac{\left(t-t_{0}\right)^{2}}{\tau}}
$$


Figure 2 Optimization results for the best (left, smallest least-square deviation) and worst (right, largest least-square deviation) fitted models.

The solid lines represent the model and the points depict the measured data. Note that there are no measurements for $z$ (hidden node of the network). 
which is a suitable model for presumption (2). Adding the non-linear term $-\beta_{H} y^{3}$ to the right-hand side, an amplitude-dependent frequency may be realized by the ODE:

$\ddot{y}=-\left(\left(2 \pi \omega_{H}\right)^{2}+\frac{2}{\tau}+4\left(\frac{\left(t-t_{0}\right)^{2}}{\tau}\right)\right) y-\frac{t-t_{0}}{\tau} \dot{y}-\beta_{H} y^{3}$,

$y(0)=y_{0}, \dot{y}(0)=\nu_{0}$.

Mutual coupling of both components yields a network of two nodes:

$\ddot{x}=-\left(2 \pi \omega_{L}\right)^{2} x-\mu \dot{x}-\beta_{L} x^{3}-\varepsilon_{x y} y$

$\ddot{y}=-\left(\left(2 \pi \omega_{H}\right)^{2}+\frac{2}{\tau}+4\left(\frac{\left(t-t_{0}\right)^{2}}{\tau}\right)\right) y-\frac{t-t_{0}}{\tau} \dot{y}-\beta_{H} y^{3}-\varepsilon_{y x} x$,

$x(0)=x_{0}, \quad \dot{x}(0)=\chi, \quad y(0)=y_{0}, \quad \dot{y}(0)=\nu_{0}$.

Thus, the model is determined by a set of nine parameters $\left(\omega_{L}, \omega_{H}, \mu, \beta_{L}, \beta_{H}, \tau, t_{0}, \varepsilon_{x y}, \varepsilon_{y x}\right)$ and four initial values. The model was fitted to a burst pattern with a duration of $2 \mathrm{~s}$ using least-square deviation from the model to the measured data points as the error criterion. Then $x$ was fitted to original burst data extracted as described above. There are no direct measurements of the high-frequency component $y$. Thus, the whole EEG was bandpass filtered (FIR filter, order 256, pass band $8-12 \mathrm{~Hz}$ ) and the resulting signal proportion was used to fit the second model component $y$, as shown in the example in Figure 3.

One possible application is to track the parameters within a BSP, since the sedation depth varies in this phase. If changes in sedation depth result in differences in burst pattern, such changes should be visible in parameter alterations. We investigated a sequence of consecutive bursts in a patient and identified the parameter vector $\left(\omega_{L}, \omega_{H}, \mu, \beta_{L}, \beta_{H}, \tau, t_{0}, \varepsilon_{x y}, \varepsilon_{y x}\right)$ in a preliminary study. By analyzing the results of individual cases, the following hypotheses can be formulated (an example is shown in Figure 4):

1. The time course of $\beta_{L}$ is characterized by a short rise at the beginning of a BSP and a slow depression at the end of burst suppression.

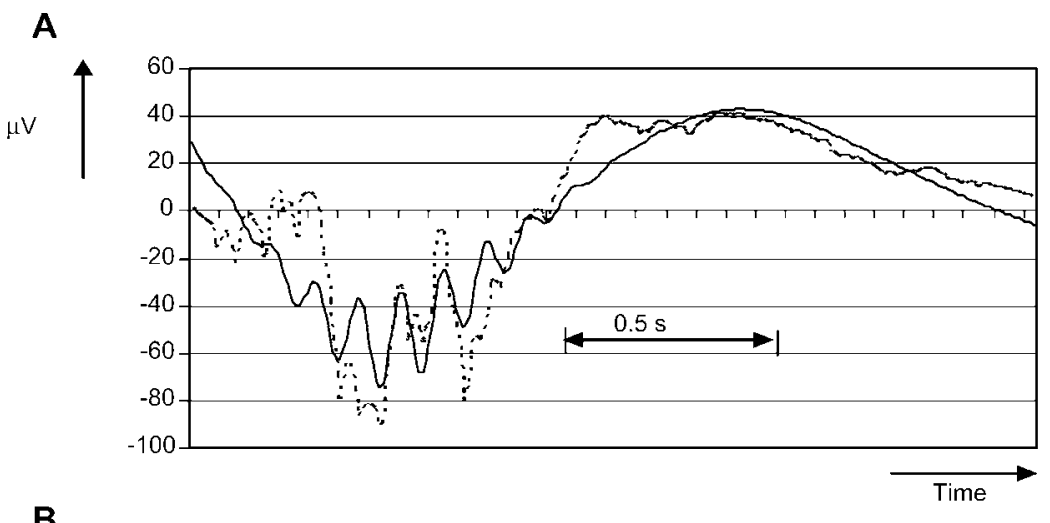

B
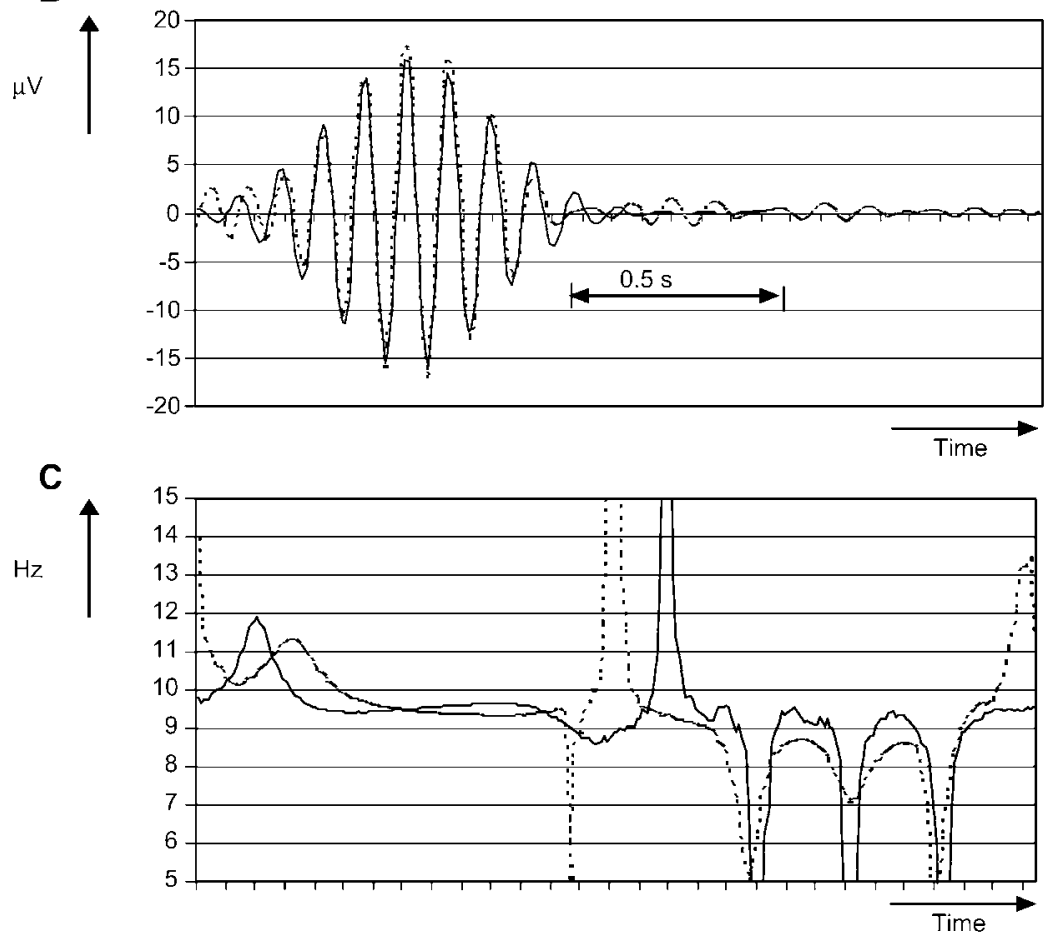

Figure 3 Results of modeling of a representative burst pattern.

(A) Original (dashed line) and modeled (full line) burst pattern. (B) Extracted spindle-like component of the original and modeled burst patterns (filtering at 8-12 Hz). (C) Instantaneous frequency courses of both spindle-like components. 
$\omega$


$t_{0}$

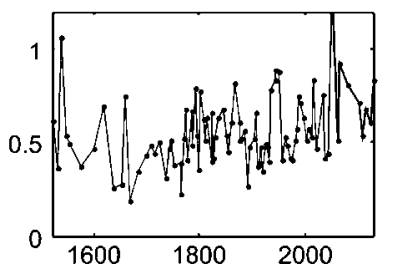

$\beta_{L}$

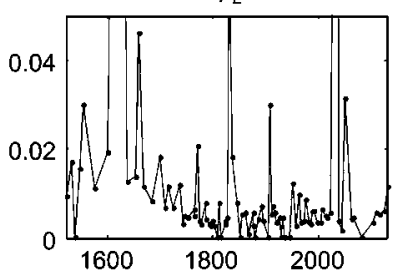

$\omega_{H}$

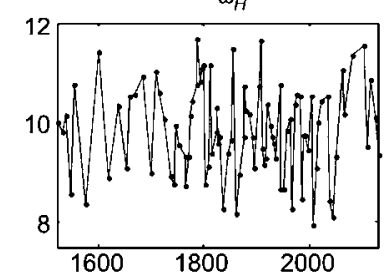

$\beta_{H}$

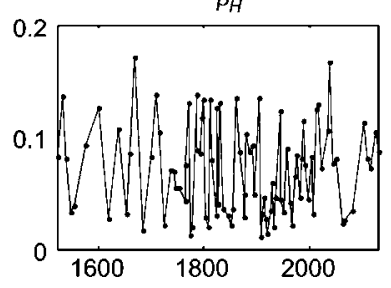

$\mu$

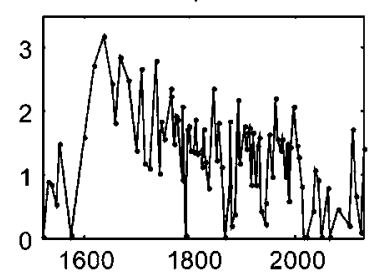

$\tau$
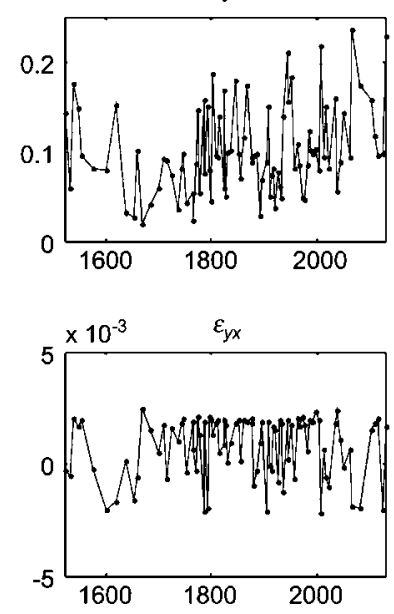

Figure 4 Parameter development during a burst-suppression period.

Each burst pattern immediately following a suppression period was modeled and the parameter set identified was marked. The time scale is given in seconds.

2. Analogously, the time course of $\mu$ is characterized by a short rise at the beginning of a BSP and a slow depression at the end of burst suppression.

3. The parameter $\tau$ increases in the course of burst suppression, that is, the spindles appear to be broader.

4. The parameter $t_{0}$ increases in the course of burst suppression, that is, the spindles occur later with respect to the low-frequency negative half-wave.

Further investigations in a separate study should examine how these trends may be statistically confirmed.

\section{Discussion and conclusion}

Oscillatory phenomena in electrical brain activity can be understood within the framework of coupled oscillator theory [21]. This is also true for the cooperative dynamics of oscillatory components of, e.g., the cardiovascularrespiratory system [3]. As already demonstrated by other studies [3, 6, 9, 14, 24], simulations of physiological systems using coupled oscillator systems and corresponding model-based analysis methods not only significantly contribute to improved interpretation of results, but also enhance the analysis methods themselves. For quantification and interpretation of complex coupling patterns, such simulations represent an indispensable processing step. The overview of the types of modeling and simulation strategies presented here, as well as their applications, demonstrates the major advantages of our strategy.

Limitations should be noted, however. The modeling is time-consuming and the parameters of the ODE systems are difficult to interpret. These limitations are possibly due to the fact that the model is too simplistic. Yet the advantages are clear and significant. The examples derived from our applications fully demonstrate the important clinical relevance of these model-related approaches.

\section{Acknowledgements}

This work was supported by Deutsche Forschungsgemeinschaft (DFG grants Wi 1166/2-3/4, Wi 1166/6-1/2 and Ha 2899/3-1).

\section{References}

[1] Arnold M, Witte H, Schelenz C. Time-variant investigation of quadratic phase couplings caused by amplitude modulation in electroencephalic burst-suppression patterns. J Clin Monit Comput 2002; 17: 115-123.

[2] Bock HG. Numerical treatment of inverse problems in chemical reaction systems. Springer Ser Chem Phys 1981; 18: 102-125.

[3] Cimponeriu L, Rosenblum MG, Fieseler T, et al. Inferring asymmetric relations between interacting neuronal oscillators. Prog Theor Phys Suppl 2003; 150: 22-36.

[4] de Boer RW. Beat-to-beat blood pressure fluctuations and heart rate variability in man: physiological relationships, analysis techniques and a simple model. Ph.D thesis, University of Amsterdam 1985.

[5] Eiselt M, Schindler J, Arnold M, Witte H, Zwiener U, Frenzel J. Functional interactions within the newborn brain investigated by adaptive coherence analysis of EEG. Neurophysiol Clin 2001; 31: 104-113.

[6] Glass L. Synchronization and rhythmic processes in physiology. Nature 2001; 410: 277-284.

[7] Gobbele R, Buchner H, Curio G. High-frequency $(600 \mathrm{~Hz})$ SEP activities originating in the subcortical and cortical 
human somatosensory system. Evoked potentials. Electroencephalogr Clin Neurophysiol 1998; 108: 182-189.

[8] Gobbele R, Waberski TD, Simon H, et al. Different origins of low- and high-frequency components $(600 \mathrm{~Hz})$ of human somatosensory evoked potentials. Clin Neurophysiol 2004; 115: 927-937.

[9] Grasman J, Jansen M. Mutually synchronized relaxation oscillators as prototypes of oscillating systems in biology. J Math Biol 1979; 7: 171-197.

[10] Gray CM. Synchronous oscillations in neuronal systems: mechanism and functions. J Comput Neurosci 1994; 1: 11-38.

[11] Kirkpatrick S, Gelatt CD, Vecchi MP. Optimization by simulated annealing. Science 1983; 220: 671-680.

[12] Leistritz L, Jäger H, Schelenz C, et al. New approaches for detection and analysis of electroencephalographic burstsuppression patterns in patients with sedation. J Clin Monit 1999; 15: 357-367.

[13] Leistritz L, Suesse T, Haueisen J, Hilgenfeld B, Witte H. Methods for parameter identification in oscillatory networks and application to cortical and thalamic $600 \mathrm{~Hz}$ activity. J Physiol Paris 2006; 99: 58-65.

[14] Linkens D, Taylor I, Duthie H. Mathematical modeling of the colorectal myoelectric activity in humans. IEEE Trans Biomed Eng 1976; 23: 101-110.

[15] Marquardt D. An algorithm for least squares estimation on nonlinear parameters. SIAM J Appl Math 1963; 11: 431-441.

[16] Nielson HB. Damping parameter in Marquardt's method. Technical report IMM-REP-1999-05. Department of Mathematical Modeling, Technical University of Denmark 1999.

[17] Sarkela M, Mustola S, Seppanen T, et al. Automatic analysis and monitoring of burst suppression in anesthesia. $J$ Clin Monit Comput 2002; 17: 125-134.
[18] Schack B, Witte H, Helbig M, Schelenz C, Specht M. Timevariant non-linear phase-coupling analysis of EEG burst patterns in sedated patients during electroencephalic burst suppression period. Clin Neurophysiol 2001; 112: 1388-1399.

[19] Singer W, Gray CM. Visual feature integration and the temporal correlation hypothesis. Annu Rev Neurosci 1995; 18 : 555-586.

[20] Solis FJ, Wets RJB. Minimization by random search techniques. Math Operat Res 1981; 6: 19-30.

[21] Stefanovska A, Luchinsky DG, McClintock PVE. Modelling couplings among oscillators of the cardiovascular system. Physiol Meas 2001; 22: 551-564.

[22] Wang DL. Object selection based on oscillatory correlation. Neural Netw 1999; 12: 579-592.

[23] Witte H, Putsche P, Eiselt M, et al. Analysis of the interrelations between a low-frequency and a high-frequency signal component in human neonatal EEG during quiet sleep. Neurosci Lett 1997; 236: 175-179.

[24] Witte $\mathrm{H}$, Rother M. Better quantification of neonatal respiratory sinus arrhythmia-progress by modelling and model-related physiological examinations. Med Biol Eng Comput 1989; 27: 289-306.

[25] Witte H, Schack B, Helbig M, et al. Quantification of transient quadratic phase couplings within EEG burst patterns in sedated patients during electroencephalic burst-suppression period. J Physiol Paris 2000; 94: 427-434.

[26] Witte $H$, Schelenz $C$, Specht $M$, et al. Interrelations between EEG frequency components in sedated intensive care patients during burst-suppression period. Neurosci Lett 1999; 260: 53-56.

[27] Witte $H$, Schelenz $C$, Specht $M$, et al. Interrelations between EEG frequency components in sedated intensive care patients during burst-suppression period. Neurosci Lett 1999; 260: 53-56. 